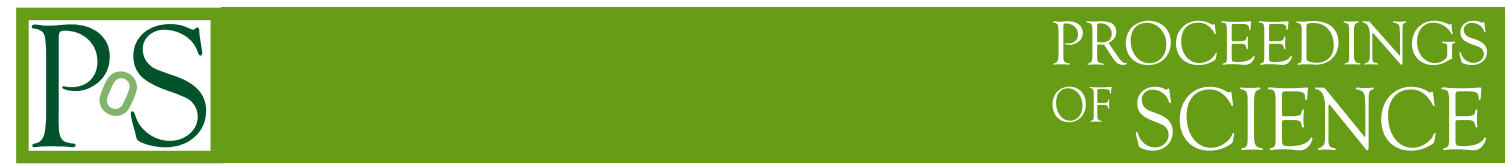

\title{
Kaon physics at KLOE and KLOE-2 prospects
}

\section{Bloise ${ }^{* \dagger}$}

Frascati Laboratory of INFN, Italy

E-mail: 'caterina.bloise@lnf. infn.it!

The KLOE experiment has measured most of the decay branching fractions (BR) of $\mathrm{K}_{S}, \mathrm{~K}_{L}$ and $\mathrm{K}^{ \pm}$mesons, providing precision results on the CKM parameter $\mathrm{V}_{u s}$, and the most sensitive unitarity test of the quark flavor mixing matrix. We are presently finalizing i) the analysis of the $\mathrm{BR}\left(\mathrm{K}^{ \pm} \rightarrow \pi^{+} \pi^{+} \pi^{-}\right)$that completes the measurements of the kaon dominant BR's, and ii) the update of the upper limit on the $\mathrm{BR}\left(\mathrm{K}_{S} \rightarrow 3 \pi^{0}\right)$, published in year 2005. The neutral kaon system offers the unique opportunity to perform tests of CPT invariance and quantum mechanics (QM). In particular, a new analysis of the KLOE data is in progress to test CPT and Lorentz symmetries in the framework of the Standard-Model Extension (SME), exploiting the EPR correlation in the neutral kaon pairs produced at the $\phi$-factory. I report on the ongoing analyses and point out the prospects for further improvements in sensitivity on kaon physics with the KLOE-2 experiment, that has been approved to integrate of order of $20 / \mathrm{fb}$ with an upgraded detector.

The 2011 Europhysics Conference on High Energy Physics, EPS-HEP 2011,

July 21-27, 2011

Grenoble, Rhône-Alpes, France

\footnotetext{
*Speaker.

$\dagger$ On behalf of the KLOE and KLOE-2 Collaboration
} 


\section{1. $\mathbf{V}_{u s}$}


suring the main BRs of $K_{L}, K^{ \pm}$, and $K_{S}$ (unique to KLOE), including semileptonic and two-body decays; lifetime measurements for $K_{L}, K_{S}[\overline{2}]$ and $K^{ \pm}$; form factor slopes from the analysis of $K_{L} e 3$ and $K_{L} \mu 3$. These data together with the value of $\left|V_{u s}\right| /\left|V_{u d}\right|$ from the measurement of the $K^{ \pm} \rightarrow \mu^{ \pm} v(\gamma)$ branching ratio and the extraction of $\left|V_{u d}\right|$ from superallowed nuclear $\beta$ decays, provide the basis for testing the unitarity of the quark-flavor mixing matrix. To extract $\left|V_{u s}\right| \times f_{+}(0)$ we use the $S U(2)$-breaking and long-distance $E M$ corrections to the radiation-inclusive decay rate [3].]. The measured values of $\left|V_{u s}\right| \times f_{+}(0)$ from the five semileptonic decay modes agree well within the errors and average to $\left|V_{u s}\right| \times f_{+}(0)=0.2157 \pm 0.0006$, with $\chi^{2} / n d f=7.0 / 4$ (Prob=13\%). The $0.28 \%$ accuracy of this result has to be compared with the $0.23 \%$ of the world average $\left|V_{u s}\right| \times f_{+}(0)$ $=0.2166 \pm 0.0005$ [A-1]. Using the determination of $\left|V_{u s}\right| \times f_{+}(0)$ from $K_{l 3}$ decays and the value $f_{+}(0)=0.964(5)$ from ['; has been obtained from the comparison of the radiation-inclusive decay rates of $K^{ \pm} \rightarrow \mu^{ \pm} v(\gamma)$ and $\pi^{ \pm} \rightarrow \mu^{ \pm} v(\gamma)$, combined with lattice calculation of $f_{K} / f_{\pi}$ [遂]. Using $\operatorname{BR}\left(K^{ \pm} \rightarrow \mu^{ \pm} v\right)=$

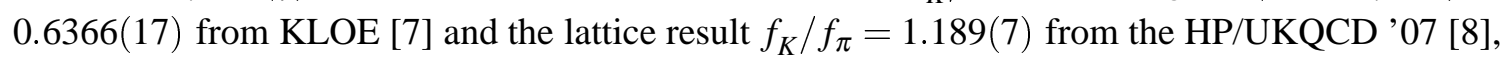
we obtain $\left|V_{u s}\right| /\left|V_{u d}\right|=0.2323(15)$. This value has been used in a fit together with the measure-

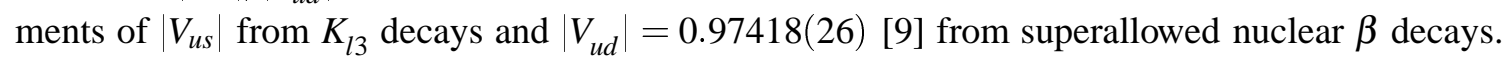
The result is $\left|V_{u d}\right|=0.97417(26)$ and $\left|V_{u s}\right|=0.2249(10)$, from which CKM unitarity is confirmed through $\Delta_{C K M}=1-\left(\left|V_{u d}\right|^{2}+\left|V_{u s}\right|^{2}+\left|V_{\mathrm{ub}}\right|^{2}\right)=4(7) \times 10^{-4}$. The sensitivity of the quark-lepton universality test is competitive with the measurements from $\tau$ decays and from the electroweak


$\gamma \gamma$ tagger system, the inner tracker based on the C-GEM technology, and the calorimeters at low polar angle, have been approved for integrating, in year 2012-2015, 20/fb at DA $\phi \mathrm{NE}$ that is adopting a new craib-waist beam interaction scheme to increase the luminosity to $\sim 5 \times 10^{32} \mathrm{~cm}^{-2} \mathrm{~s}^{-1}$. KLOE-2 can significantly improve the accuracy on the measurement of $K_{L}, K^{ \pm}$lifetimes and $K_{S} e 3$ branching ratio with respect to present world average [1, 121$]$, reducing the $0.23 \%$ fractional uncertainty on $\left|V_{u s}\right| \times f_{+}(0)$ to one per mil level. Lattice QCD simulations have recently made tremendous progress and in near-term the accuracy on $f_{+}(0)$ could reach the experimental sensitivity on $\left|V_{u s}\right| \times f_{+}(0)$. At that level, the precision test of CKM unitarity, $\sigma\left(\Delta_{C K M}\right)=2-3 \times 10^{-4}$, will be limited by the accuracy on $\left|V_{u d}\right|$ presently at $0.026 \%$ [1] $\left.{ }_{1}^{1} \overline{3}\right]$ and dominated by the uncertainty on radiative corrections.

\section{2. $\mathbf{K}^{+} \rightarrow \pi^{+} \pi^{+} \pi^{-}$}

The $\mathrm{BR}\left(\mathrm{K}^{+} \rightarrow \pi^{+} \pi^{+} \pi^{-}\right)$was obtained with $4 \%$ relative precision and a new measurement at $0.5 \%$ level has an impact on the semileptonic decays (thus on $\left|V_{u s}\right| \times f_{+}(0)$ ) and on the $\pi \pi$ scattering lengths from the $\mathrm{K}^{ \pm} \rightarrow \pi^{ \pm} \pi^{0} \pi^{0}$ cusp analysis. The ongoing study of the $\mathrm{K}^{+} \rightarrow \pi^{+} \pi^{+} \pi^{-}$ decay is based on i) the selection of $\mathrm{K}^{+}$candidates (tagging procedure) by the identification of $\mathrm{K}^{-} \rightarrow \pi^{-} \pi^{0}$ and $\mathrm{K}^{-} \rightarrow \mu^{-} v$ samples, independently treated; ii) the reconstruction of the $\mathrm{K}^{+}$path from the kinematical constraints given by the $\mathrm{K}^{-}$momentum and $\phi$ momentum (from Bhabhascattering events); ii) the backward extrapolation of any charged track not belonging to the $\mathrm{K}^{-}$ 
decay chain; iii) the reconstruction of the track closest-approach point $\left(\mathbf{C A}_{i}\right)$ and closest-approach distance $\left(\mathrm{CAd}_{i}\right)$ to the $\mathrm{K}^{+}$path; iv) the selection of events with at least two tracks with $\mathrm{CAd}_{i} \leq 3 \mathrm{~cm}$ and $\mathbf{C A}_{i}$ outside the drift chamber (DC) (for a better control of systematics from tagging procedure); v) the measurement of the missing-mass distribution, $\mathrm{M}_{\text {miss }}^{2}=\Delta^{2} \mathrm{E}_{K^{+}-\pi \pi}-\left|\Delta \mathbf{P}_{K^{+}-\pi \pi}\right|^{2}$. The tagging procedure affects the selection efficiency of the $\mathrm{K}^{+} \rightarrow \pi^{+} \pi^{+} \pi^{-}$sample introducing a bias (tag-bias) on the BR measurement that has been evaluated by Monte Carlo simulation and controlled by the comparison of the two tagging channels. A further control of the tag-bias is in progress, by the selection of a third tagging sample based on the $\mathrm{K}^{-}$identification through the $\mathrm{dE} / \mathrm{dx}$ measurement in the DC. The selection efficiency of the $\mathrm{K}^{+} \rightarrow \pi^{+} \pi^{+} \pi^{-}$sample $(\sim 7 \%)$ is obtained from the study of a control sample of $\mathrm{K}^{+} \rightarrow \pi^{-} X$. A fit to the missing-mass distribution with the Monte Carlo shapes for signal and residual backgrounds (1\% level of contamination by other decays almost uniformly distributed in the $\mathrm{M}_{\text {miss }}^{2}$ range of interest) is used for the event counting. From the preliminary analysis of a subsample of $174 / \mathrm{pb}$ we obtained a relative error on the absolute BR of $0.6 \%$ that we are checking to assess the various contributions.

3. $\mathbf{K}_{S} \rightarrow \pi^{0} \pi^{0} \pi^{0}$

The $C P$-violating transition $K_{S} \rightarrow \pi^{0} \pi^{0} \pi^{0}$ is expected with a BR of $1.70(2) \times 10^{-9}$. KLOE has obtained the upper limit $\mathrm{BR}\left(K_{S} \rightarrow 3 \pi^{0}\right) \leq 1.2 \times 10^{-7}$ at $90 \%$ C.L. using a sample of 450/pb [i1 4íl]. The main background source is given by incorrectly reconstructed $K_{S} \rightarrow \pi^{0} \pi^{0}$ decays, when the photon-energy deposit in the calorimeter is reconstructed as split clusters, or additional photons from machine background are assigned to the $K_{S}$ decay. The result, a factor of ten better than the previous upper limit, has allowed KLOE to increase the sensitivity on the $C P$ and $C P T$ parameters using the unitary condition (Bell-Steinberger relation) as explained in Ref. [1] $1 \overline{1} \overline{1} \overline{1}]$. Since then, further improvements on the clustering procedure to recover erroneously-split photon clusters are proven to reduce contamination by a factor of six while leaving the signal efficiency unaffected. The ongoing analysis proceeds through i) the selection of $K_{S}$ candidates by the identification of $K_{L}$ interactions in the calorimeter requiring an energy release $\Delta \mathrm{E}_{K}>129 \mathrm{MeV}$ and $0.196<\beta_{K}<0.250$; ii) the selection of events with six prompt neutral clusters (not connected to any track in the DC and with times compatible with the hypothesis of photons from the interaction region); iii) the $\chi_{\text {evt }}^{2}$ evaluation with the measurements of the position, energy and time of each photon constrained by total energy-momentum conservation: the events with $\chi_{\text {evt }}^{2}>35$ are rejected; iv) the evaluation of $\chi_{3 \pi}^{2}$ and $\chi_{2 \pi}^{2}$ with the measurements of the position, energy and time of each photon in the hypothesis of $K_{S} \rightarrow 3 \pi^{0}$, and $K_{S} \rightarrow 2 \pi^{0}$ plus two additional clusters from machine background, respectively. The candidates are those with $\chi_{3 \pi}^{2} \leq 4.6$ and $12 \leq \chi_{2 \pi}^{2} \leq 60$; v) the rejection of events that in the second hypothesis at point iv have a total energy $\operatorname{Etot}_{2 \pi}>\left(510 \mathrm{MeV}-1.7 \sigma_{E}\right)$; vi) the evaluation of the minimal distance among photon clusters in the event, keeping as candidates those with $\mathrm{R}_{\min }>65 \mathrm{~cm}$. The selection efficiency is obtained from Monte Carlo simulation, $\varepsilon_{3 \pi}=0.19(1)$. From the selected sample at point i) the $K_{S} \rightarrow 2 \pi^{0}$ events are also identified $\left(\varepsilon_{2 \pi}=0.660(3)\right)$ and used as normalization for the $\operatorname{BR}\left(K_{S} \rightarrow 3 \pi^{0}\right)$ measurement. No candidate survives the analysis selection, and $9.0 \times 10^{7} K_{S} \rightarrow 2 \pi^{0}$ decays are found in the tagged sample. The preliminary result for the upper limit, on 1.7/fb of integrated luminosity, is $\mathrm{BR}\left(K_{S} \rightarrow 3 \pi^{0}\right)<2.910^{-8}$ at $90 \%$ C.L., a factor of 4 better than the previous KLOE result. Studies are in progress to evaluate the increase 
in acceptance at KLOE-2, from the installation of the crystal calorimeters at low angle [1] $\left.{ }_{1}^{1} \overline{1}\right]$, and the improvement in tagging efficiency from the addition of $K_{L}$ decay samples. This is important, together with the background suppression obtained by the ongoing analysis, in the perspective of a first evidence of the process at KLOE-2.

\section{QM and CPT test with neutral kaon interferometry}

Several tests of QM and CPT invariance have been performed at KLOE, where time evolution of the neutral kaon pairs is sensitive to decoherence phenomena, and to the breakdown of CPT symmetry dealt with in the standard Lorentz-symmetry-violating Extension of the Standard Model (SME) [1] 1 ind. The first evidence of the interference has been obtained in year 2006. Since then, more data have been analyzed and improvements in the analysis procedures have brought the results on decoherence and CPT-violating parameters presented in Ref.[1] $\left.{ }_{1}\right]$. An analysis of the $K_{S} K_{L} \rightarrow \pi^{+} \pi^{-} \pi^{+} \pi^{-}$final state is in progress to improve on the SME parameters, through the measurement of the decay amplitude as a function of the delay between kaon decays, ordered according to the quadrant in the celestial coordinate frame. The reconstruction of events in the region $\Delta \mathrm{t} \sim 0$ is crucial for the precision measurement of the interference term and the inner tracker, that is being constructed for the KLOE-2 experiment, will improve the resolution from $\sigma_{\Delta t} \sim \tau_{S}$ to $\sim 0.3 \tau_{S}$, and the sensitivity to most of the QM and CPT tests by a factor of 2 . The physics reach on kaon interferometry at KLOE-2, up to a factor of ten better than present data, is



\section{References}

[1] F. Bossi, E. De Lucia, J. Lee-Franzini, S. Miscetti, M. Palutan (KLOE), Riv. Nuovo Cim. 031, 531 (2008)

[2] F. Ambrosino et al. (collaboration for the KLOE), Eur.Phys.J. C71, 1604 (2011), 1011.2668

[3] V. Cirigliano, M. Giannotti, H. Neufeld, JHEP 11, 006 (2008), 0807 . 4507

[4] F. Ambrosino et al. (KLOE), JHEP 04, 059 (2008), 0802.3009

[5] P.A. Boyle et al., Phys. Rev. Lett. 100, 141601 (2008), 0710.5136

[6] W.J. Marciano, Phys. Rev. Lett. 93, 231803 (2004), hep-ph / 0402299

[7] F. Ambrosino et al. (KLOE), Phys. Lett. B632, 76 (2006), hep-ex/ 0509045

[8] E. Follana, C. Davies, G.P. Lepage, J. Shigemitsu (HPQCD), Phys. Rev. Lett. 100, 062002 (2008)

[9] I.S. Towner, J.C. Hardy, Phys. Rev. C77, 025501 (2008), 0710 . 3181

[10] W.J. Marciano, PoS KAON, 003 (2008)

[11] F. Archilli (KLOE-2 Collaboration), PoS ICHEP2010, 482 (2010)

[12] G. Amelino-Camelia, F. Archilli, D. Babusci, D. Badoni, G. Bencivenni et al., Eur.Phys.J. C68, 619 (2010)

[13] M. Antonelli, D.M. Asner, D.A. Bauer, T.G. Becher, M. Beneke et al., Phys.Rept. 494, 197 (2010)

[14] F. Ambrosino et al. (KLOE), Phys. Lett. B619, 61 (2005), hep-ex/ 0505012

[15] F. Ambrosino et al. (KLOE), JHEP 12, 011 (2006), hep-ex/ 0610034

[16] A. Di Domenico et al. (KLOE), J. Phys. Conf. Ser. 171, 012008 (2009) 